\title{
On a mechanism low-pressure insertion of chain molecules into crystalline matrices.
}

\author{
E.V. Vakarin, J.P. Badiali \\ LECA ENSCP-UPMC-CNRS, 11 rue P. et M. Curie, 75231 Cedex 05, Paris, France
}

\begin{abstract}
A microscopic mechanism of low-pressure insertion and separation of chain-like molecules in host matrices is proposed. It is shown that the intramolecular correlations combined to appropriate host activities are responsible for a low-pressure condensation of chain molecules. This allows recover a fine structure of the isotherms and to explain recent experiments on the insertion of $\mathrm{C}_{2} \mathrm{H}_{2}$ and $\mathrm{CO}_{2}$ guest species. We argue that the mechanism should be dominant in low-dimensional host geometries, where the entropic effects are strongly suppressed and the major factors are the chain connectivity and packing.
\end{abstract}

\section{INTRODUCTION}

Insertion of guest species into host matrices has recently attracted a considerable interest as a designing tool for hydrogen storage systems [1], rechargeable batteries and superconducting devices [2]. Recent progress in synthesis of new composite materials $[3,4]$ opens novel perspectives in separation and storage of larger molecules (e.g., acetylene, methane or even longer hydrocarbons) which have important technological applications. In particular, a highly controlled acetylene accommodation in a metal-organic microporous material has recently been reported [5]. It has been found that due to formation of regular hydrogen bonds the guest molecules are arranged into dense onedimensional strips at very low relative pressures. This allows for a safe storage at a density much higher than the compression limit of free acetylene. In contrast, $\mathrm{CO}_{2}$ isotherms exhibit a much weaker adsorption in this pressure domain. This offers an efficient way of separation of these two compounds. In this context it has been emphasized [6] that the fabrication of high-capacity adsorbents, sensitive to particular guest species, requires a better knowledge of the host-guest and guest-guest interactions. In particular, our understanding of a microscopic mechanism, that allows to reach high densities inside a host marix at very low bulk pressures, would be quite helpful for a designing of novel storage and separation systems.

In this Letter we report on a possible microscopic mechanism, responsible for a remarkable enhancement in the insertion of chain-like molecules into crystalline host matrices at low bulk pressures. The main idea is to note that in the host-guest contact the guest acts as a thermodynamic subsystem that has an impact (energetic and entropic) from its bulk. This induces a correlation for the guest species, such that their accommodation is not driven only by the accessibility of the matrix space but also by effective (density-dependent) interactions, arising from the packing and the chain connectivity effects. For planar geometries this mechanism has already found experimental evidences $[7,8]$ and interesting kinetic interpretations $[9]$.

\section{INSERTION MODEL}

The host is modeled as a lattice of adsorbing sites with the lattice spacing $d$ and coordination number $q$. The lattice is assumed to be absolutely rigid, such that possible guest-induced distortions [10] or restructurings [11] are excluded. The guest molecule is modeled as a chain (length $m$ ) of hardsphere segments [12] (diameter $\sigma$ ). In this way we take into account the chain connectivity and the excluded volume effects. Therefore, the guest fluid is characterized by its bulk density $\rho$ (or packing $\left.\eta=\pi \rho \sigma^{3} / 6\right)$ and the chain length $m$. The latter is the number of hard sphere segments which mimic the functional groups (e.g. $m=2$ for $C_{2} H_{2}$ ). The bulk equation of state can be approximated as [12]

$$
\frac{\beta P}{\rho}=\frac{\beta P_{h s}}{\rho}-\frac{m-1}{m}\left(1-\frac{\eta}{2-\eta}+\frac{3 \eta}{1-\eta}\right)
$$

where $P_{h s}$ is the hard sphere contribution and $\beta=1 /(k T)$ is the inverse temperature. In what follows this equation of state will be used to make a link between the bulk density $\rho$ and the pressure $P$.

The adsorption potential $U_{s}\left(\mathbf{r}_{i}\right)$ is given by the sticky site model $[13,14]$

$$
\exp \left(-\beta U_{s}\left(\mathbf{r}_{i}\right)\right)=1+\lambda \sum_{\mathbf{R}_{m}} \delta\left(\mathbf{r}_{i}-\mathbf{R}_{m}\right)
$$

where $\mathbf{r}_{i}$ is a chain segment position, $\mathbf{R}_{m}$ is a position of a lattice site and $\lambda$ is the stickiness parameter. The latter can be related to the segment adsorption energy $\epsilon$ through $\lambda=\exp (\beta \epsilon)$. This singular one-body potential allows one to perform an exact integration in the partition function

$$
Z=Z_{\text {ref }} \sum_{n=0} \frac{\lambda^{n}}{n !} \sum_{\left\{\mathbf{R}_{k}\right\}} \rho_{n}^{r e f}\left(\mathbf{R}_{1}, \ldots, \mathbf{R}_{n}\right)
$$


where $Z_{r e f}$ is the partition function for the same system but without the adsorbing potential, and $\rho_{n}^{r e f}\left(\mathbf{R}_{1}, \ldots, \mathbf{R}_{n}\right)$ is the $n$-body distribution function taken at positions of the lattice sites. In general, even in the absence of a specific adsorption, a periodic host structure induces a density redistribution and particle correlations around the lattice strands. Nevertheless, in a low-pressure regime (on which focus), these effects can be neglected and the reference state can be approximated by the bulk state. Therefore, we have in eq. (3) an infinite series on $\lambda$ including the correlations of all orders for the reference state. If only pair correlations are important then the problem can be mapped onto the lattice gas model $[13,14]$

$$
\Xi=Z / Z_{\text {ref }}=\sum_{\left\{t_{i}\right\}} \exp \left(-\beta H_{L G}\right)
$$

with the Hamiltonian

$$
H_{L G}=\sum_{i j} W\left(\mathbf{R}_{i}, \mathbf{R}_{j}\right) t_{i} t_{j}-\sum_{i} \mu\left(\mathbf{R}_{i}\right) t_{i}
$$

in which $\left\{t_{i}\right\}$ is a set of occupation numbers. The effective chemical potential $\mu\left(\mathbf{R}_{i}\right)$ and pair interaction $W\left(\mathbf{R}_{i}, \mathbf{R}_{j}\right)$ are closely connected with the properties of the fluid in the bulk phase. Namely $[13,14]$,

$$
\begin{gathered}
\beta \mu\left(\mathbf{R}_{i}\right)=\ln \left(\lambda \rho_{1}^{r e f}\left(\mathbf{R}_{i}, \sigma / 2\right)\right) \\
\beta W\left(\mathbf{R}_{i}, \mathbf{R}_{j}\right)=-\ln \left(g_{2}^{r e f}\left(\mathbf{R}_{i}, \mathbf{R}_{j}\right)\right)
\end{gathered}
$$

where $\rho_{1}^{r e f}\left(\mathbf{R}_{i}\right)$ and $g_{2}^{r e f}\left(\mathbf{R}_{i}, \mathbf{R}_{j}\right)$ are respectively the one-body and pair correlation functions for the reference state (i.e., a state without specific adsorption). The latter is independent of the adsorption site position because the lattice is translationally invariant. If $\sigma \approx d$ then we deal with the contact values of the strand-segment density profile $\rho_{1}(\sigma / 2)$ and the segment-segment correlation function $g_{2}(\sigma)$. The contact density is approximated by its hard wall-chain value [14]

$$
\rho_{1}(\sigma / 2)=\rho \sigma^{3}\left[\frac{1+2 \eta}{(1-\eta)^{2}}-\frac{m-1}{m} \frac{1}{1-\eta}\right]
$$

Here the second term describes a chain depletion compared to a purely monomeric case $(m=1)$. At low pressures (bulk densities) this effect is marginal and $\rho_{1}(\sigma / 2)$ can be replaced by $\rho \sigma^{3} / m$ in this domain. In the Percus-Yevick approximation the pair correlation is given by [15]

$$
g_{2}(\sigma)=\frac{1+\eta / 2}{(1-\eta)^{2}}-\frac{m-1}{m} \frac{Q}{1-\eta}+\left[\frac{m-1}{m}\right]^{2} \frac{K}{\eta}
$$

Here the first term corresponds to the hard sphere packing effects which are dominating with increasing $\eta$. The second term describes a depletion with increasing $m$ due to the chain-chain repulsion the intermolecular correlation. The last term in $g_{2}(\sigma)$ is responsible for the intramolecular correlation inside a chain. The latter is dominating at low densities when the chain-chain contacts are rather rare and the segments correlate mainly through the chain connectivity. That is, a probability to find two randomly chosen segments in the same chain is rather high. The singularity at $\eta \rightarrow 0$ is a flaw of the Percus-Yevick approximation, which, however, does not affect our conclusions. The coefficients $K$ and $Q$ control the corresponding magnitudes, which depend on the chemical nature of molecules (e.g. chain flexibility) and on the dimensionality of space (in 1D, for instance, the intermolecular correlations are realized only through the chain ends). Note that the chain length $m$ is chosen as an external variable. This means that we do not consider the clustering process itself, assuming that the guest cluster composition remains fixed in the course of insertion. This assumption is valid if the adsorption is not dissociative or if the adsorption rate dominates the rate of dissociation in the fluid bulk.

\section{A. Effective interaction}

As we have already noticed, due to correlations in the reference state (bulk), the adsorbed guest species experience an effective pairwise lateral interaction $\beta W=-\ln g_{2}(\sigma)$, where $g_{2}(\sigma) \approx g_{2}(d)$ is given by eq. (9). In Figure 1 this quantity is analyzed as function of the packing $\eta$ at different chain lengths $m$. It is seen that $\beta W$ is a non-monotonic function. In a low-density (pressure) domain, dominated by the intramolecular correlations, the interaction is strongly attractive. This effect increases with the chain length. At intermediate densities the chain-chain competition for the available space (the intermolecular correlations) leads to an effective repulsion at neighboring lattice sites. Finally, at high densities the chain connectivity plays only a marginal role since a closely packed chain configuration becomes identical to that of disconnected hard-sphere segments. This leads to an effective attraction [13,14], similar to the depletion forces in colloidal systems. Although our analysis is based on the approximate result eq. (9), our conclusions should hold in general because, from the experimental point of view [7-9], the density/concentration regimes (e.g. diluted, semidiluted and plateau regimes) are clearly distinguished. 


\section{B. Insertion isotherms}

Having determined the inputs to the lattice gas Hamiltonian we can find the mean-field approximation for the free energy, which gives the adsorption isotherm $[13,14]$

$$
\theta=\frac{\lambda \rho_{1}(\sigma / 2)\left[g_{2}(\sigma)\right]^{q \theta}}{1+\lambda \rho_{1}(\sigma / 2)\left[g_{2}(\sigma)\right]^{q \theta}}
$$

where $\theta=\left\langle\sum_{i} t_{i}\right\rangle / N$ is the coverage - the guest density inside the matrix. Equation (10) has been solved numerically in order to determine the coverage $\theta$ as a function of density $\eta$ and chain length size $m$. Then a pressure dependence can be obtained through the equation of state (1). The result for a dimeric fluid $(m=2)$ on a four-fold coordinated lattice $(q=4)$ is plotted in Figure 2. It is seen that the isotherm shape is coherent with the density (pressure) modulation of the effective interaction $\beta W$ (see Figure 1). Namely, at low pressures when the chain-chain contacts are negligible, the adsorption of one bead promotes the adsorption of the entire chain through the intramolecular correlations. As a result we have a remarkable increase of the guest density $\theta$ in a very narrow pressure domain. This cooperative effect has been confirmed experimentally [7]. At intermediate pressures the chains compete for the adsorption sites (the intermolecular correlations) and the isotherm exhibits a "slower" increase with a characteristic inflection point. The latter signals a cross-over to the packing regime, where the isotherm reaches a saturation. It is remarkable that the overall shape of the isotherm very closely resembles the one reported for acetylene [5], including the lowpressure feature (see the inset to Figure 1(a) in that paper) signalling a very efficient accommodation. On the other hand, the $\mathrm{CO}_{2}$ molecules (which are of similar size as $\mathrm{C}_{2} \mathrm{H}_{2}$ and can also be viwed as diatomics) do not exhibit this effect. The chain length difference could explain the different adsorption behavior as a segregation phenomenon [16], even without considering a specific adsorption.

Therefore, we have to find out why the chains of the same length could behave differently. With this purpose we have analyzed the parameters, at which the low-pressure condensation occurs. We have studied the solution of eq. (10) at $m=2$ and one-dimensional (1D) $(q=2)$ geometry that mimics a channeled host structure, reported in [5]. Interactions in the neighboring channels were neglected and the in-channel interactions were assumed to be governed by $g_{2}(\sigma)$. A fit to the experimental data [5] is presented in Figure 3. It seen that the theory is qualitatively consistent with the data. It is found that the low-pressure feature sharpens with increasing stickiness $\lambda$ (segment binding energy).
In order to be free from possible artifacts of the mean-field approximation we have studied an exact solution for $1 \mathrm{D}$ geometry [17]

$$
M=\frac{\sinh (H)}{\sqrt{\sinh ^{2}(H)+e^{4 J}}},
$$

where $J=\beta W, H=\beta(\mu-W) / 2, M=2 \theta-1$. It is found that there are two regimes (see the inset in Figure 3). In a low-activity regime ( $\operatorname{small} \lambda$ ) the isotherm exhibits a gradual adsorption without the low-density condensation, while in the high-activity regime (large $\lambda$ ) we observe this feature. This can explain a striking difference between $\mathrm{C}_{2} \mathrm{H}_{2}$ and $\mathrm{CO}_{2}$ [5]. Although the latter can also be viewed as diatomic (possibly with different inter- and intra-molecular constants $Q$ and $K$ ), its interaction with the host is much weaker that that of $\mathrm{C}_{2} \mathrm{H}_{2}$, which is found [5] to form two hydrogen bonds with non-coordinated oxygens in the host matrix.

In conclusion, we have found that an interplay of the inter- and intramolecular correlations combined to a variation in the host activity is an efficient tool for low-pressure insertion and separation of chain molecules. The proposed mechanism should be dominant in low-dimensional geometries, where the entropic effects (due to the chain conformation and reorientation) are strongly suppressed. We argue that the mechanism is not exclusive to particular guest molecules, such that the $\mathrm{C}_{2} \mathrm{H}_{2}$ insertion should be considered as a prototypic example. Therefore, a detailed study of a correlation between the host geometry, activity and the chain length and architecture could open novel perspectives in the storage and separation of other technologically important species. In particular, a proper control of the guest-induced matrix distortions [10] or restructuring [11] could lead to a suitable matching or mismatching between the host geometry and the guest architecture. This offers a promising tool for swithching between the highand low-activity regimes, increasing the insertion selectivity in a given domain of controlling thermodynamic parameters (e. g. pressure, density, concentration).

[1] L. Schlapbach, A. Zuttel, Nature (London), 2001, 414,353

[2] J. S. Slusky et al., Nature (London), 2001, 410, 343

[3] O. M. Yaghi et al., Nature (London), 2003, 423, 705

[4] G. Ferey et al., Acc. Chem. Res. 2005, 38, 217 
[5] R. Matsuda et al. Nature (London), 2005, 436, 238

[6] G. Ferey, Nature (London), 2005, 436, 187

[7] A. Voronov, I. Luzinov, S. Minko, A. Sidorenko, E. Vakarin, M. Holovko, Macromolecules 30, 6929 (1997)

[8] A. Voronov, E. Pefferkorn, S. Minko, Macromolecules 31, 6387 (1998)

[9] S. Minko, A. Voronov, E. Pefferkorn, Langmuir 2000, 16, 7876

[10] E. V. Vakarin, J. P. Badiali, M. D. Levi, D. Aurbach, Phys. Rev. B 2001, 63, 014304

[11] E. V. Vakarin, J. P. Badiali, J. Phys. Chem. B 2002, 106, 7721

[12] M. S. Wertheim, J. Chem. Phys. 1987, 87, 7323

[13] J. P. Badiali, L. Blum, M. L. Rosinberg, Chem. Phys. Lett. 129, 149 (1986)

[14] E. V. Vakarin, Yu. Duda, M. F. Holovko, J. Chem. Phys. 107, 5569 (1997)

[15] J. Chang, S. I. Sandler, J. Chem. Phys. 102, 437 (1995)

[16] E. V. Vakarin, J. Chem. Phys. 109, 338 (1998)
[17] R. J. Baxter, Exactly Solvable Models in Statistical Mechanics ( Academic Press, NY, 1982)

FIG. 1. The effective lateral interaction $\beta W$ as a function of the bulk density $\eta$ at different chain lengths $m$.

FIG. 2. Mean-field adsorption isotherm for dimers $m=2$ on a lattice with coordination $q=4$. Other parameters are $\lambda=800, K=0.15, Q=2$. The saturation pressure $P_{0}$ corresponds to $\theta \rightarrow 1$. The inset shows typical chain arrangements and correlations in different pressure domains.

FIG. 3. Fitting of the mean-field isotherm (line) to the experimental data of Ref.[5] (symbols) for $\mathrm{C}_{2} \mathrm{H}_{2}$ insertion. The saturation pressure $P_{0}$ was taken to be $5 k P a$. The inset shows the exact $1 \mathrm{D}$ isotherms at different activity regimes (see text). 


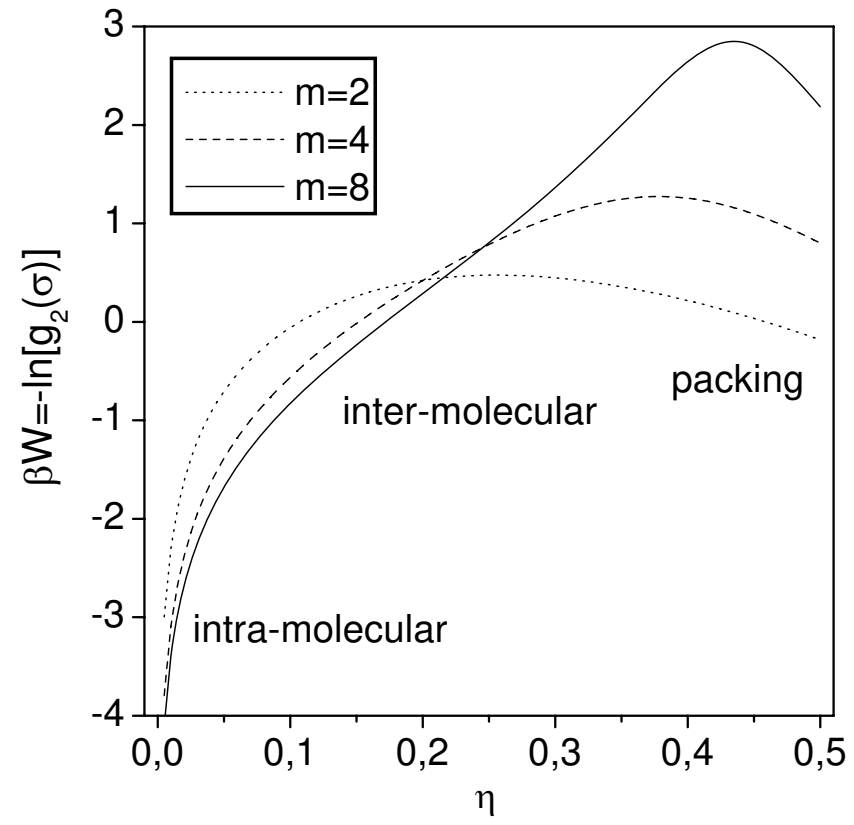

Figure1 


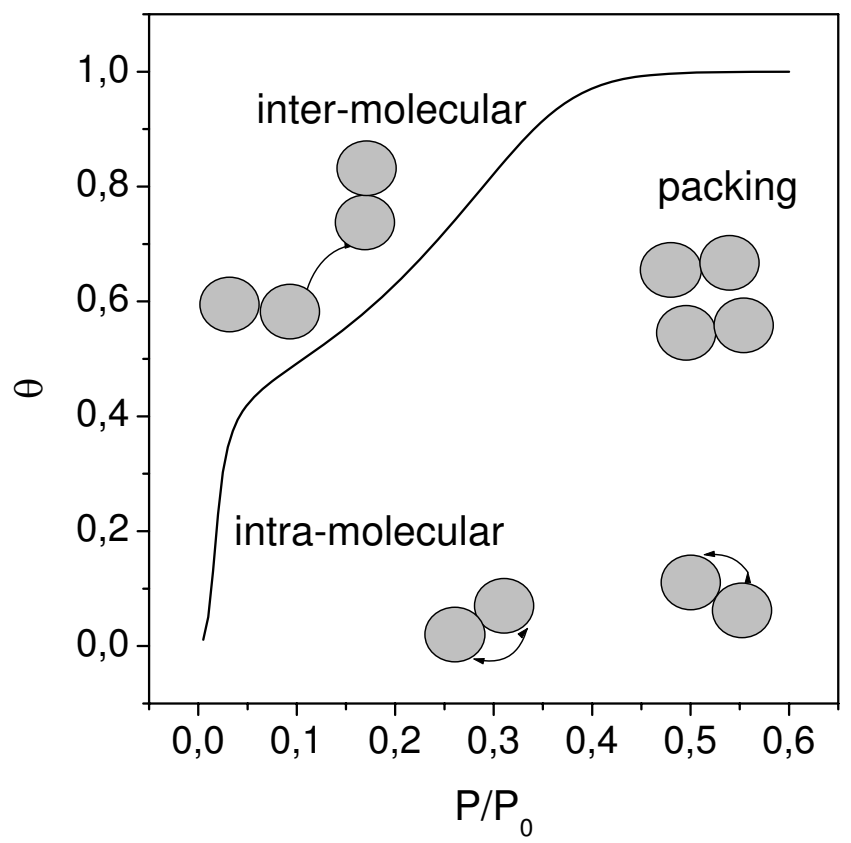

Figure 2 


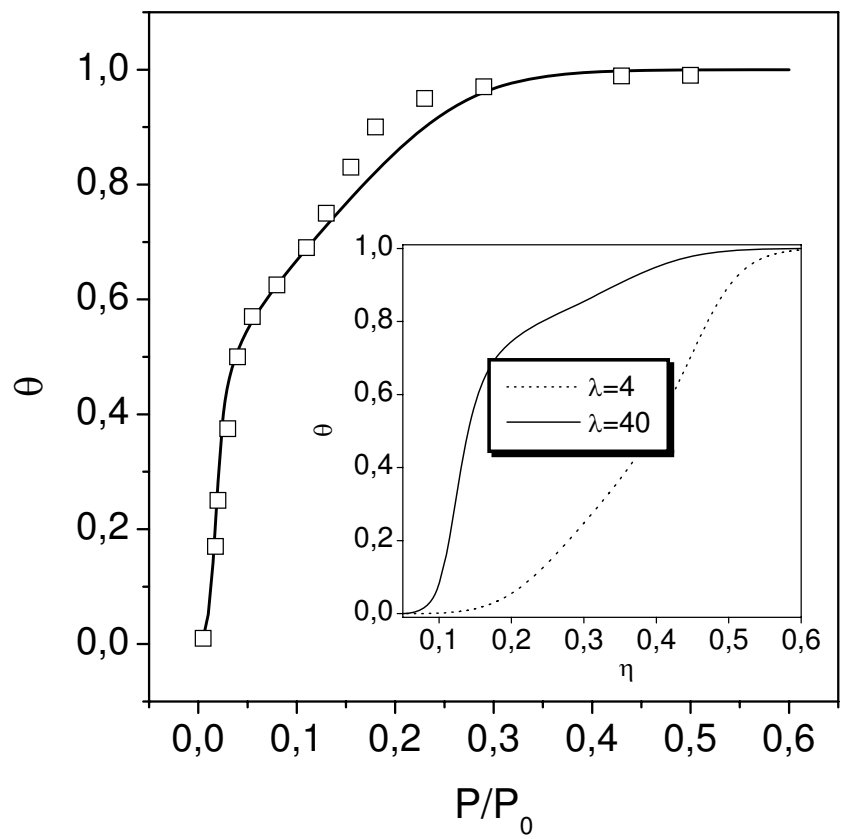

Figure 3 